\title{
A New Iterative Method for CT Reconstruction with Uncertain View Angles
}

\author{
Riis, Nicolai André Brogaard; Dong, Yiqiu
}

Published in:

Proceedings of7th International Conference on Scale Space and Variational Methods in Computer Vision

Link to article, DOI:

10.1007/978-3-030-22368-7_13

Publication date:

2019

Document Version

Peer reviewed version

Link back to DTU Orbit

Citation $(A P A)$ :

Riis, N. A. B., \& Dong, Y. (2019). A New Iterative Method for CT Reconstruction with Uncertain View Angles. In J. Lellmann, J. Modersitzki, \& M. Burger (Eds.), Proceedings of7th International Conference on Scale Space and Variational Methods in Computer Vision (pp. 156-167). Springer. Lecture Notes in Computer Science (including subseries Lecture Notes in Artificial Intelligence and Lecture Notes in Bioinformatics) Vol. 11603 LNCS https://doi.org/10.1007/978-3-030-22368-7_13

\section{General rights}

Copyright and moral rights for the publications made accessible in the public portal are retained by the authors and/or other copyright owners and it is a condition of accessing publications that users recognise and abide by the legal requirements associated with these rights.

- Users may download and print one copy of any publication from the public portal for the purpose of private study or research.

- You may not further distribute the material or use it for any profit-making activity or commercial gain

- You may freely distribute the URL identifying the publication in the public portal 


\title{
A New Iterative Method for CT Reconstruction with Uncertain View Angles
}

\author{
Nicolai André Brogaard Riis ${ }^{(凶)}$ and Yiqiu Dong \\ Department of Applied Mathematics and Computer Science, Technical University \\ of Denmark, Richard Petersens Plads, 2800 Kongens Lyngby, Denmark \\ nabr@dtu.dk
}

\begin{abstract}
In this paper, we propose a new iterative algorithm for Computed Tomography (CT) reconstruction when the problem has uncertainty in the view angles. The algorithm models this uncertainty by an additive model-discrepancy term leading to an estimate of the uncertainty in the likelihood function. This means we can combine state-of-theart regularization priors such as total variation with this likelihood. To achieve a good reconstruction the algorithm alternates between updating the CT image and the uncertainty estimate in the likelihood. In simulated $2 \mathrm{D}$ numerical experiments, we show that our method is able to improve the relative reconstruction error and visual quality of the $\mathrm{CT}$ image for the uncertain-angle CT problem.
\end{abstract}

Keywords: Computed Tomography $\cdot$ Uncertain view angles ·

Model error · Variational methods · Total variation •

Model discrepancy

\section{Introduction}

In this paper, we consider Computed Tomography (CT) where the geometry of the physical set-up is only known approximately. The goal is to achieve reconstructions that are stable in the presence of uncertainties in the geometric parameters. We restrict our attention to uncertainty in the view angles. We assume that the actual view angles are realizations of some known probability distribution $\pi_{\text {angles }}(\cdot)$ and that the measured sinogram is corrupted by additive Gaussian noise with known mean and covariance.

With the above assumptions, we formulate the $\mathrm{CT}$ reconstruction problem under uncertain view angles as estimating the unknown attenuation coefficient image $\mathbf{x} \in \mathbb{R}^{n}$ from a measured (noisy) sinogram $\mathbf{b} \in \mathbb{R}^{m}$ following the model

$$
\mathbf{b}=\mathbf{A}(\boldsymbol{\theta}) \mathbf{x}+\mathbf{e}, \quad \boldsymbol{\theta} \sim \pi_{\text {angles }}(\boldsymbol{\theta}), \mathbf{e} \sim \mathcal{N}\left(\boldsymbol{\mu}_{\mathbf{e}}, \mathbf{C}_{\mathbf{e}}\right),
$$

The work was supported by the National Natural Science Foundation of China via Grant 11701388.

(C) Springer Nature Switzerland AG 2019

J. Lellmann et al. (Eds.): SSVM 2019, LNCS 11603, pp. 156-167, 2019.

https://doi.org/10.1007/978-3-030-22368-7_13 
where $\mathbf{e} \in \mathbb{R}^{m}$ is the additive Gaussian noise with mean $\boldsymbol{\mu}_{\mathbf{e}}$ and symmetric positive definite covariance $\mathbf{C}_{\mathbf{e}}$. The parameterized matrix $\mathbf{A}(\boldsymbol{\theta}) \in \mathbb{R}^{m \times n}$ is the discrete approximation of the Radon transform with view angles $\boldsymbol{\theta} \in \mathbb{R}^{q}$. The measurement at detector element $l$ from the view angle $\theta_{i}$ with $i=1, \ldots, q$, i.e., $\left(\mathbf{A}\left(\theta_{i}\right) \mathbf{x}\right)_{l}$, is a discretization of $(\mathcal{R} f)\left(\theta_{i}, \mathrm{~s}_{l}\right)=\int_{\mathbb{R}} f\left(\mathrm{~s}_{l} V\left(\theta_{i}\right)+t V^{\perp}\left(\theta_{i}\right)\right) \mathrm{d} t$, where $\mathrm{s}_{l}$ gives the position of the $l$ th pixel on the detector with $l=1, \cdots, p$, and $m=q p$. Moreover, the function $f$ is the continuous representation of $\mathbf{x}$, $\mathrm{V}(\theta)=(\cos \theta, \sin \theta)$ and $\mathrm{V}^{\perp}(\theta)=V(\theta+\pi / 2)$. For more details on the mathematical model of CT see e.g. [1]. We emphasize that the goal in this work is to estimate the CT image $\mathbf{x}$ from a measured sinogram $\mathbf{b}$ according to the model (1) with uncertain view angles $\boldsymbol{\theta}$ and noise e. Here, $\boldsymbol{\theta}$ and $\mathbf{e}$ are considered as nuisance or uninteresting parameters, and they are only taken into account when reconstructing $\mathbf{x}$ without being explicitly estimated.

\subsection{Previous Work}

Many variational methods have been proposed for CT reconstruction, see e.g., [2-4]. In general, variational models in these methods consist of a data-fitting term and a regularization term, and these two terms are balanced by a regularization parameter. In order to deal with the ill-posedness in CT reconstruction problems, the choice of regularization is very important. Different regularization techniques have been applied, for example, total variation (TV) regularization [5] and framelet representations [2]. But these methods do not take parametric uncertainty such as uncertainty in view angles into account. Therefore, good performance of the methods is not guaranteed if the view angles are uncertain.

The CT reconstruction problem with uncertain view angles in (1) is generally solved by estimating the view angles from some measurements. Geometrical calibration of models in CT has been studied, see e.g. [6] for a review. Typically such methods are based on reference objects or reference instruments for the calibration. Recently, in the case of uncertain or unknown view angles a few reconstruction methods that only use the measured sinogram without reference objects or instruments have been proposed, see [7-9]. These methods aim to estimate the view angles $\boldsymbol{\theta}$ in addition to the CT image $\mathbf{x}$, and can be categorized into two groups: (1) Estimating view angles directly from projection data and then estimating the CT image and (2) simultaneously estimating view angles and CT image.

In [9] it has been shown that if the scanned objects are asymmetrical then view angles can be uniquely determined by sinogram measurements. According to this result, we can estimate angles directly from complete measurements. However, if the object is partly symmetrical or the measurements are not sufficient, we cannot obtain an accurate angle estimation, see [8]. Then, due to error propagation, an inaccurate angle estimation would lead to an unsatisfactory reconstruction.

The simultaneous methods such as Bayesian sampling-based methods [7] can effectively avoid error propagation, but they are limited by computational complexity and generally require many evaluations of the forward model (1), which makes them unfeasible for large-scale problems. 
There are also a few methods for characterizing and reducing model errors in general inverse problems, see [10-15]. Most of these methods are based on the statistical description of the model error in a Bayesian setting. This leads to a natural way of incorporating uncertainties and modelling errors in the model. However, full Bayesian methods also suffer from computational complexity issues except in cases when the object is assumed to follow a Gaussian distribution, in which case closed-form solutions exist.

\subsection{Our Contribution}

In this paper, we propose a new iterative algorithm for CT reconstruction with uncertain view angles. The main step in the algorithm is based on a variational method, which combines the state-of-the-art regularization such as TV with a modified data-fitting term, that includes the uncertainty in the view angles via a model-discrepancy term. Since the model-discrepancy term depends on the estimated reconstruction, we update it and the reconstruction alternately. The simulated numerical results show that the new algorithm is able to reduce the relative error and improve the visual quality of the reconstructions.

\section{Our Method}

The CT reconstruction with uncertain view angles is formulated in (1) with an assumption of the probability distribution on the view angles. By including the known expected view angles, $\hat{\boldsymbol{\theta}}$, we can reformulate the problem as follows.

$$
\mathbf{b}=\mathbf{A}(\hat{\boldsymbol{\theta}}) \mathbf{x}+\boldsymbol{\eta}+\mathbf{e}, \quad \mathbf{e} \sim \mathcal{N}\left(\boldsymbol{\mu}_{\mathbf{e}}, \mathbf{C}_{\mathbf{e}}\right),
$$

where the new random variable $\boldsymbol{\eta}=\boldsymbol{\eta}(\boldsymbol{\theta}, \mathbf{x})=\mathbf{A}(\boldsymbol{\theta}) \mathbf{x}-\mathbf{A}(\hat{\boldsymbol{\theta}}) \mathbf{x}$ with $\boldsymbol{\theta} \sim$ $\pi_{\text {angles }}(\boldsymbol{\theta})$ models the uncertainties associated with the view angles. Note that (2) is consistent with (1). By this reformulation, we basically shift the uncertainties in the view angles to the model-discrepancy term $\boldsymbol{\eta}$, which will be used to derive our variational model.

Defining modelling errors as an additive model-discrepancy term was first applied in [16] in the field of model calibration of physical and computer models. The distribution of $\boldsymbol{\eta}$ was assumed as a Gaussian Process and determined as a model correction term in addition to $\mathbf{x}$. In $[11,17]$, this idea was applied in Bayesian inverse problems and named as the Approximation Error Approach (AEA). The main differences are that in the AEA $\boldsymbol{\eta}$ is used to represent the difference between two grid systems instead of a model discrepancy and it is marginalized out in the likelihood function. The outputs of the AEA are the distributions of $\mathbf{x}$ and $\boldsymbol{\eta}$. To further improve the results, in [14] an iterative scheme was introduced where the distributions of $\mathbf{x}$ and $\boldsymbol{\eta}$ are updated alternately. 
Inspired by the ideas of the AEA, we derive the likelihood according to the model (2) by marginalizing out both $\boldsymbol{\eta}$ and e. Define $\boldsymbol{\nu}=\boldsymbol{\eta}+\mathbf{e}$, and the likelihood is given by

$$
\pi(\mathbf{b} \mid \mathbf{x})=\int_{\mathbb{R}^{m}} \pi(\mathbf{b}, \boldsymbol{\nu} \mid \mathbf{x}) \mathrm{d} \boldsymbol{\nu}=\int_{\mathbb{R}^{m}} \pi(\mathbf{b} \mid \mathbf{x}, \boldsymbol{\nu}) \pi(\boldsymbol{\nu} \mid \mathbf{x}) \mathrm{d} \boldsymbol{\nu}=\pi_{\boldsymbol{\nu} \mid \mathbf{x}}(\mathbf{b}-\mathbf{A}(\hat{\boldsymbol{\theta}}) \mathbf{x} \mid \mathbf{x}) .
$$

The conditional distribution of $\boldsymbol{\nu} \mid \mathbf{x}$ may be rather complicated, but we can approximate it by a simpler distribution such as a Gaussian. Gaussian approximations has been shown experimentally to be reasonable in many applications $[11,13,14,17,18]$. Here, we assume that $\boldsymbol{\eta} \mid \mathbf{x}$ follows a Gaussian distribution $\mathcal{N}\left(\boldsymbol{\mu}_{\boldsymbol{\eta} \mid \mathbf{x}}, \mathbf{C}_{\boldsymbol{\eta} \mid \mathbf{x}}\right)$ with mean $\boldsymbol{\mu}_{\boldsymbol{\eta} \mid \mathbf{x}}$ and covariance $\mathbf{C}_{\boldsymbol{\eta} \mid \mathbf{x}}$ and $\mathbf{e}$ is independent of $\mathbf{x}$. Then we obtain the negative log-likelihood function

$$
-\log \pi(\mathbf{b} \mid \mathbf{x}) \propto \frac{1}{2}\left\|\mathbf{b}-\mathbf{A}(\hat{\boldsymbol{\theta}}) \mathbf{x}-\boldsymbol{\mu}_{\boldsymbol{\nu} \mid \mathbf{x}}\right\|_{\mathbf{C}_{\nu \mid \mathbf{x}}^{-1}}^{2}=\frac{1}{2}\left\|\mathbf{L}_{\boldsymbol{\nu} \mid \mathbf{x}}\left(\mathbf{b}-\mathbf{A}(\hat{\boldsymbol{\theta}}) \mathbf{x}-\boldsymbol{\mu}_{\boldsymbol{\nu} \mid \mathbf{x}}\right)\right\|_{2}^{2}
$$

where $\boldsymbol{\mu}_{\boldsymbol{\nu} \mid \mathbf{x}}=\boldsymbol{\mu}_{\mathbf{e}}+\boldsymbol{\mu}_{\boldsymbol{\eta} \mid \mathbf{x}}, \mathbf{C}_{\boldsymbol{\nu} \mid \mathbf{x}}=\mathbf{C}_{\mathbf{e}}+\mathbf{C}_{\boldsymbol{\eta} \mid \mathbf{x}}$ is the combined covariance of the measurement noise and model discrepancy, and $\mathbf{L}_{\boldsymbol{\nu} \mid \mathbf{x}}^{T} \mathbf{L}_{\boldsymbol{\nu} \mid \mathbf{x}}=\mathbf{C}_{\boldsymbol{\nu} \mid \mathbf{x}}^{-1}$ is the Cholesky factorization of the inverse covariance. Applying regularization techniques, we can formulate a variational model for (2) that gives a stable solution with respect to the uncertain view angles and measurement noise using the likelihood (4). TV regularization has shown good performance in CT [2], and thus we use it as regularization term and obtain the following variational model

$$
\min _{\mathbf{x} \geq 0} \frac{1}{2}\left\|\mathbf{L}_{\boldsymbol{\nu} \mid \mathbf{x}}\left(\mathbf{b}-\mathbf{A}(\hat{\boldsymbol{\theta}}) \mathbf{x}-\boldsymbol{\mu}_{\boldsymbol{\nu} \mid \mathbf{x}}\right)\right\|_{2}^{2}+\lambda \mathrm{TV}(\mathbf{x})
$$

with minimizer $\mathbf{x}_{\mathrm{STV}}$ and regularization parameter $\lambda>0$. We use $\operatorname{TV}(\mathbf{x})=$ $\sum_{i}\left\|[\nabla \mathbf{x}]_{i}\right\|_{2}$, where $\left\|[\nabla \mathbf{x}]_{i}\right\|_{2}=\sqrt{\left(\nabla_{h} \mathbf{x}\right)_{i}^{2}+\left(\nabla_{v} \mathbf{x}\right)_{i}^{2}}$, with $\left(\nabla_{h} \mathbf{x}\right)_{i}$ and $\left(\nabla_{v} \mathbf{x}\right)_{i}$ denoting the derivatives of $\mathbf{x}_{i}$ along horizontal and vertical directions with symmetric boundary condition, respectively. A non-negativity constraint is added because the attenuation coefficients $\mathbf{x}$ cannot be negative.

\subsection{An Iterative Algorithm}

The variational model defined in (5) still leaves the question of how the mean and covariance of $\boldsymbol{\eta} \mid \mathbf{x}$ are determined. Given a reconstruction $\mathbf{x}$, one can gen-

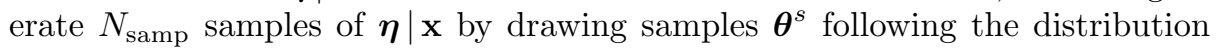
$\pi_{\text {angles }}(\boldsymbol{\theta})$, and then evaluate the model discrepancy by

$$
\boldsymbol{\eta}^{s}=\mathbf{A}\left(\boldsymbol{\theta}^{s}\right) \mathbf{x}-\mathbf{A}(\hat{\boldsymbol{\theta}}) \mathbf{x}, \quad \boldsymbol{\theta}^{s} \sim \pi_{\text {angles }}(\boldsymbol{\theta}), s=1, \ldots, N_{\text {samp }}
$$

The sample mean and covariance given $\mathbf{x}$ can then be calculated by

$$
\boldsymbol{\mu}_{\boldsymbol{\eta} \mid \mathbf{x}}^{\mathrm{samp}}=\frac{1}{N_{\mathrm{samp}}} \sum_{s=1}^{N_{\mathrm{samp}}} \boldsymbol{\eta}^{s}
$$


and

$$
\mathbf{C}_{\boldsymbol{\eta} \mid \mathbf{x}}^{\mathrm{samp}}=\frac{1}{N_{\mathrm{samp}}-1} \sum_{s=1}^{N_{\mathrm{samp}}}\left(\boldsymbol{\eta}^{s}-\boldsymbol{\mu}_{\boldsymbol{\eta} \mid \mathbf{x}}\right)\left(\boldsymbol{\eta}^{s}-\boldsymbol{\mu}_{\boldsymbol{\eta} \mid \mathbf{x}}\right)^{T} .
$$

If we have a good estimate of $\mathbf{x}$, we can obtain good samples of the model discrepancy, and then we can use the sample mean and covariance in the model (5) to further improve the reconstruction result. This leads to an iterative scheme for alternately updating the estimate of $\mathbf{x}$ and the estimates of mean and covariance of $\boldsymbol{\eta} \mid \mathbf{x}$. The iterative scheme is shown in Algorithm 1.

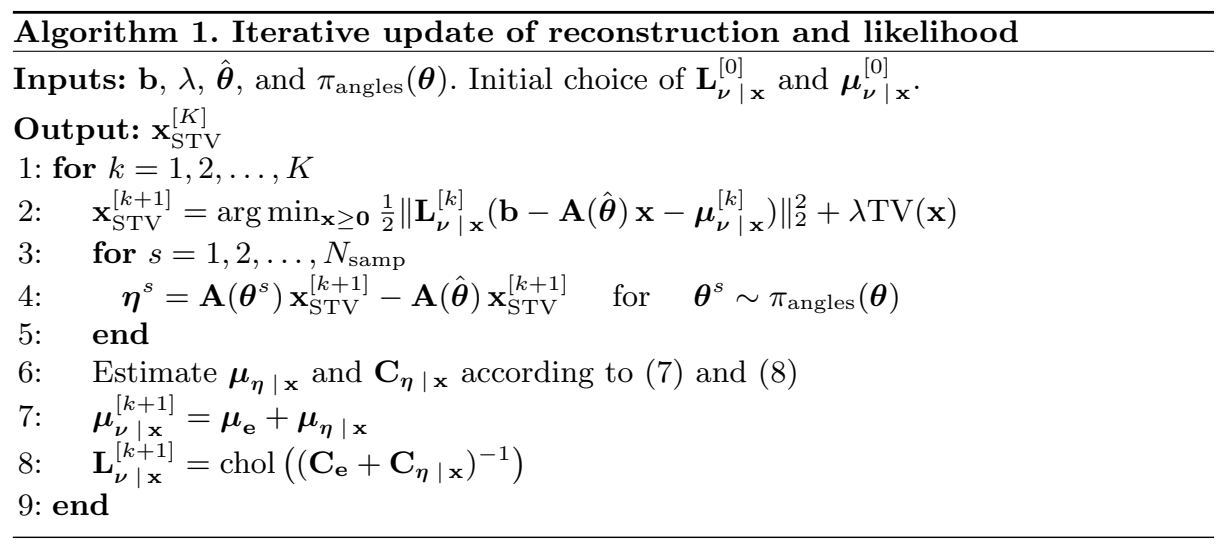

Here, $\operatorname{chol}\left(\mathbf{C}^{-1}\right)$ gives the Cholesky factorization of the inverse covariance $\mathbf{C}^{-1}$, i.e., $\mathbf{L}^{T} \mathbf{L}=\mathbf{C}^{-1}$. In the initialization, we use the measurement noise mean and covariance to initialize $\mathbf{L}_{\boldsymbol{\nu} \mid \mathbf{x}}^{[0]}=\mathbf{L}_{\mathbf{e}}$ and $\boldsymbol{\mu}_{\boldsymbol{\nu} \mid \mathbf{x}}^{[0]}=\boldsymbol{\mu}_{\mathbf{e}}$, where $\mathbf{L}_{\mathbf{e}}^{T} \mathbf{L}_{\mathbf{e}}=\mathbf{C}_{\mathbf{e}}^{-1}$.

Compared with the AEA proposed in [14], our method has two main differences. First, our method deals with the uncertainties in the model parameters and the accurate model is unknown, while in the AEA the accurate model is known and the discrepancy is between two different grid systems. Secondly, in our method, we apply a variational method incorporated with regularization techniques to obtain a reconstruction result, which can be solved by many advanced optimization methods, while in the AEA the distribution of the reconstruction is obtained by applying Bayesian inversion methods, which leads to much higher computational complexity.

\subsection{Approximation of $\mathrm{L}_{\nu \mid \mathrm{x}}$}

Because of the computational complexity in calculating the inverse and Cholesky factorization of the covariance matrix $\mathbf{C}_{\mathbf{e}}+\mathbf{C}_{\boldsymbol{\eta} \mid \mathbf{x}}$, our method can be of limited use for solving large-scale CT problems. To overcome this limitation, we 


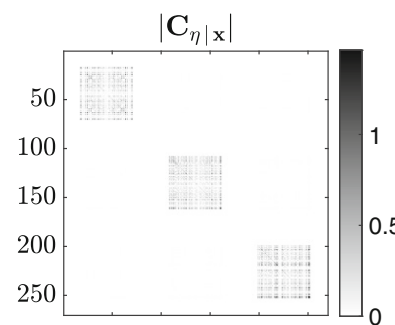

50100150200250

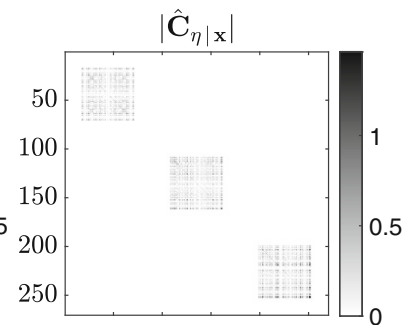

50100150200250

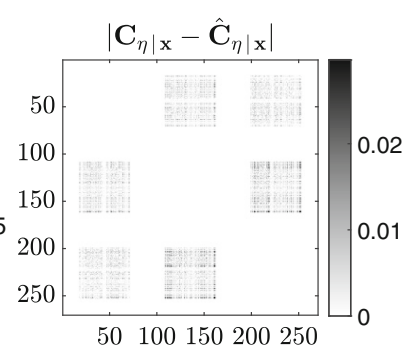

Fig. 1. An example of the absolute value of the full covariance $\mathbf{C}_{\boldsymbol{\eta} \mid \mathbf{x}}$, its approximation $\hat{\mathbf{C}}_{\boldsymbol{\eta} \mid \mathbf{x}}$ according to (9), and their absolute difference for the uncertain angle CT problem (2). We conclude that the approximation is reasonable for this problem.

approximate the covariance matrix $\mathbf{C}_{\boldsymbol{\eta} \mid \mathbf{x}} \in \mathbb{R}^{m \times m}$ by a block diagonal matrix $\hat{\mathbf{C}}_{\boldsymbol{\eta} \mid \mathbf{x}}$ given by

$$
\hat{\mathbf{C}}_{\boldsymbol{\eta} \mid \mathbf{x}}=\left[\begin{array}{llll}
\mathbf{C}_{\boldsymbol{\eta} \mid \mathbf{x}, 11} & & & \\
& \mathbf{C}_{\boldsymbol{\eta} \mid \mathbf{x}, 22} & & \\
& & \ddots & \\
& & & \mathbf{C}_{\boldsymbol{\eta} \mid \mathbf{x}, q q}
\end{array}\right]
$$

where $\mathbf{C}_{\boldsymbol{\eta} \mid \mathbf{x}, i i} \in \mathbb{R}^{p \times p}$ are the block diagonal parts of $\mathbf{C}_{\boldsymbol{\eta} \mid \mathbf{x}}, q=m / p$ is the number of view angles and $p$ is the number of detector pixels. Then, if the Gaussian measurement noise $\mathbf{e}$ is i.i.d., i.e., $\mathbf{C}_{\mathbf{e}}=\sigma^{2} \mathbf{I}_{m}$, we can compute the Cholesky factorization of the approximate inverse covariance $\left(\mathbf{C}_{\mathbf{e}}+\hat{\mathbf{C}}_{\boldsymbol{\eta} \mid \mathbf{x}}\right)^{-1}$ block-wise as follows

$$
\hat{\mathbf{L}}_{\boldsymbol{\nu} \mid \mathbf{x}}=\left[\begin{array}{lll}
\operatorname{chol}\left(\left(\mathbf{C}_{\boldsymbol{\eta} \mid \mathbf{x}, 11}+\sigma^{2} \mathbf{I}_{p}\right)^{-1}\right) & & \\
& \ddots & \\
& & \operatorname{chol}\left(\left(\mathbf{C}_{\boldsymbol{\eta} \mid \mathbf{x}, q q}+\sigma^{2} \mathbf{I}_{p}\right)^{-1}\right)
\end{array}\right]
$$

If the full covariance was used multiplication of an vector with $\mathbf{L}_{\boldsymbol{\nu} \mid \mathbf{x}}$ would require $\mathcal{O}\left(m^{2}\right)=\mathcal{O}\left(p^{2} q^{2}\right)$ operations, whereas multiplication with $\hat{\mathbf{L}}_{\boldsymbol{\nu} \mid \mathbf{x}}$ would only be $\mathcal{O}\left(p^{2} q\right)$ operations. Additionally, the matrix inversion and Cholesky factorization is reduced from $\mathcal{O}\left(m^{3}\right)=\mathcal{O}\left(p^{3} q^{3}\right)$ to $\mathcal{O}\left(p^{3} q\right)$ operations.

In Fig. 1, we show the absolute values of the full covariance $\mathbf{C}_{\boldsymbol{\eta} \mid \mathbf{x}}$, its approximation $\hat{\mathbf{C}}_{\boldsymbol{\eta} \mid \mathbf{x}}$ according to (9), and their absolute difference. The values in the off-diagonal parts are much smaller than those in the block diagonal parts of $\mathbf{C}_{\boldsymbol{\eta} \mid \mathbf{x}}$. Hence, the approximation is reasonable for this problem. In the following experiments line 8 in Algorithm 1 is therefore approximated by (10).

\section{$3 \quad$ Numerical Experiments}

In this section, we present simulated $2 \mathrm{D}$ numerical results to show the performance of our method. The experiments are carried out in MATLAB and we use 
Table 1. The physical parameters in the simulated CT experiments.

\begin{tabular}{|c|c|}
\hline Parameter & Value \\
\hline Beam type & Fan-beam \\
\hline Reconstruction domain size & $50 \mathrm{~cm} \times 50 \mathrm{~cm}$ \\
\hline Source to center distance & $50 \mathrm{~cm}$ \\
\hline Source to detector distance & $100 \mathrm{~cm}$ \\
\hline Detector length & $130 \mathrm{~cm}$ \\
\hline \multicolumn{2}{|c|}{ Small example } \\
\hline Image pixels & $n=45 \times 45$ \\
\hline Detector pixels & $p=90$ \\
\hline Number of view angles & $q=90$ \\
\hline View angle standard deviation & $\delta=1.2^{\circ}$ \\
\hline \multicolumn{2}{|c|}{ Larger example } \\
\hline Image pixels & $n=135 \times 135$ \\
\hline Detector pixels & $p=270$ \\
\hline Number of projection angles & $q=270$ \\
\hline View angle standard deviation & $\delta=0.4^{\circ}$ \\
\hline
\end{tabular}

the ASTRA toolbox [19] and "Spot Operators" [20] for matrix-free forward- and back-projections, i.e., for multiplication with $\mathbf{A}(\boldsymbol{\theta})$ and $\mathbf{A}(\boldsymbol{\theta})^{T}$. In the simulated CT problems arising from (1), the physical parameters are shown in Table 1. In both examples, the distribution of the view angles is assumed to be i.i.d. Gaussian with equidistant view angles from $0^{\circ}$ to $360^{\circ}$, denoted by $\boldsymbol{\theta}^{\text {equid }}$, as mean and $\delta^{2}$ as variance. These examples illustrate the physical case where the measurements are acquired at equidistant view angles, but each measurement is associated with some independent uncertainty. Note that the "small example" has 90 view angles and standard deviation $\delta=1.2^{\circ}$, whereas the "larger example" has 270 view angles with $\delta=0.4^{\circ}$. This is to ensure that the view angles are unlikely to switch positions relative to each other from the added uncertainty. In our numerical tests, we generate the measurements according to

$$
\mathbf{b}=\mathbf{A}\left(\boldsymbol{\theta}^{\text {machine }}\right) \overline{\mathbf{x}}+\mathbf{e},
$$

where $\overline{\mathbf{x}}$ is either the Shepp-Logan or Grains phantom generated from AIR Tools II [21], and $\boldsymbol{\theta}^{\text {machine }}$ denotes the actual view angles, which is a realization of $\mathcal{N}\left(\boldsymbol{\theta}^{\text {equid }}, \delta^{2} \mathbf{I}\right)$. Here $\mathbf{e} \sim \mathcal{N}\left(\mathbf{0}, \sigma^{2} \mathbf{I}\right)$, where $\sigma=0.005\left\|\mathbf{A}\left(\boldsymbol{\theta}^{\text {machine }}\right) \overline{\mathbf{x}}\right\|_{2} / \sqrt{m}$. We solve the TV minimization problem using the Chambolle-Pock algorithm in [22] and stopping when the relative change in the objective function is below $10^{-6}$. In our method, we set the maximum iteration number $K=10$ and the number of samples $N_{\text {samp }}=5000$. 
We compare our results with the non-negative TV reconstruction that does not take the uncertainty in the view angles into account, i.e.,

$$
\mathbf{x}_{\mathrm{TV}}=\arg \min _{\mathbf{x} \geq 0} \frac{1}{2 \sigma^{2}}\|(\mathbf{b}-\mathbf{A}(\hat{\boldsymbol{\theta}}) \mathbf{x})\|_{2}^{2}+\lambda \mathrm{TV}(\mathbf{x}),
$$

where $\hat{\boldsymbol{\theta}}=\boldsymbol{\theta}^{\text {equid }}$. In addition, we also show the results from the non-negative TV reconstruction with the actual view angles $\boldsymbol{\theta}^{\text {machine }}$, which would be the best-case scenario:

$$
\mathbf{x}_{\mathrm{TV} \text {-opt }}=\arg \min _{\mathbf{x} \geq 0} \frac{1}{2 \sigma^{2}}\left\|\left(\mathbf{b}-\mathbf{A}\left(\boldsymbol{\theta}^{\text {machine }}\right) \mathbf{x}\right)\right\|_{2}^{2}+\lambda \mathrm{TV}(\mathbf{x}) .
$$

\subsection{The Small Example}

In Fig. 2 we show the expected view angles $\hat{\boldsymbol{\theta}}=\boldsymbol{\theta}^{\text {equid }}$ and 3 realizations of $\pi_{\text {angles }}(\boldsymbol{\theta})=\mathcal{N}\left(\boldsymbol{\theta}^{\text {equid }}, \delta^{2} \mathbf{I}\right)$, i.e. 3 examples of $\boldsymbol{\theta}^{\text {machine }}$. The realizations are used to generate noisy sinograms according to (11). We compare the reconstructions $\mathbf{x}_{\mathrm{STV}}$ from our method (Algorithm 1) with the ones obtained by solving (12) and (13). In the left column of Fig. 3 we plot the relative error $\frac{\|\mathbf{x}-\overline{\mathbf{x}}\|_{2}}{\|\overline{\mathbf{x}}\|_{2}}$ of the three methods with the regularization parameter $\lambda$ varying from $10^{-6}$ to $10^{-2}$. We can see that except for large $\lambda$, where the influence of the data-fitting term becomes weak, the reconstructions from our method has lower relative errors compared to $\mathbf{x}_{\mathrm{TV}}$ from (12). With the optimal $\lambda$ choice, which gives the smallest relative error, the improvement by our method is significant. It shows the importance of taking the uncertainty in the view angles into account. In the right column of Fig. 3 we numerically show the convergence of the relative errors in our method.

In order to visually compare the reconstructions from these three methods, in Fig. 4 we show the reconstruction results for the same $\lambda$ values, which corresponds to the optimal choice in (13). It is clear that our method can effectively reduce the artifacts due to the uncertain view angles.

For this small example, in our method we can also compute the full covariance $\mathbf{C}_{\boldsymbol{\eta} \mid \mathbf{x}} \in \mathbb{R}^{m \times m}$ instead of using the approximation $\hat{\mathbf{C}}_{\boldsymbol{\eta} \mid \mathbf{x}}$ introduced in Sect. 2.2. Since the relative errors by using the full covariance are almost identical to using the approximation, we do not show them here.

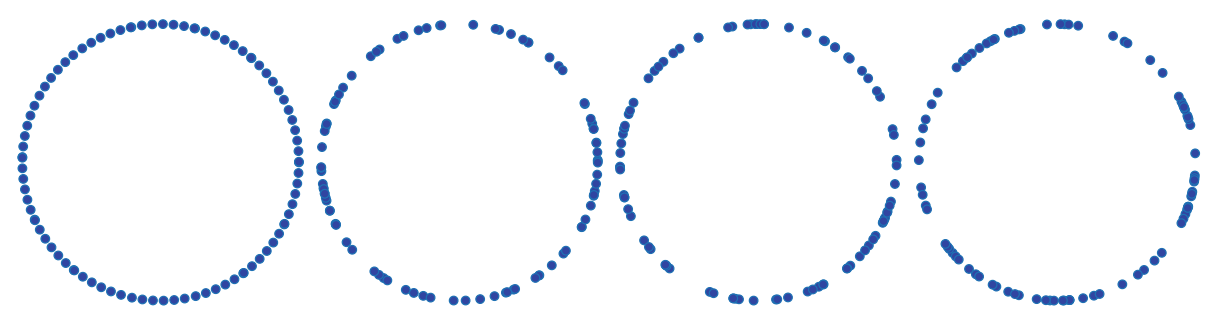

Fig. 2. For the small experiment in Table 1. From left to right: The expected view angles $\hat{\boldsymbol{\theta}}=\boldsymbol{\theta}^{\text {equid }}$ and three realizations of $\pi_{\text {angles }}(\boldsymbol{\theta})$. 

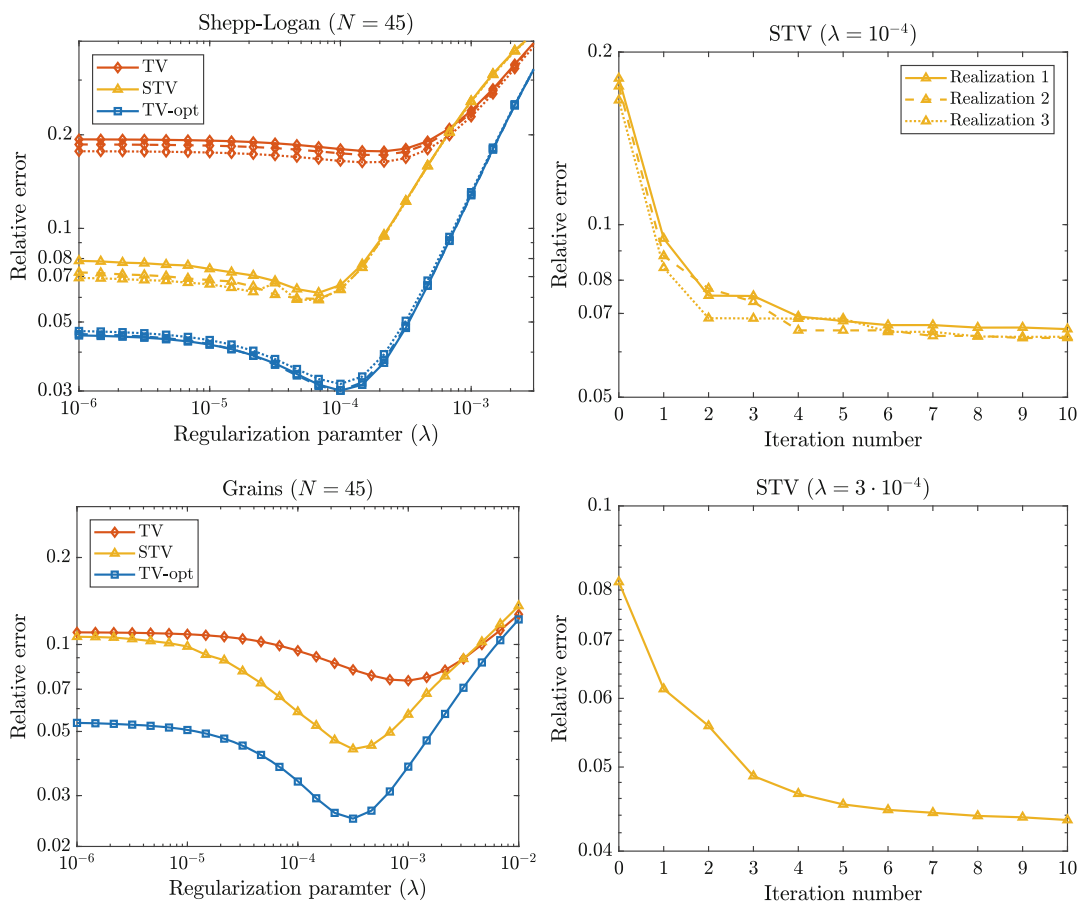

Fig. 3. For $N=45$ in Table 1. Top: Shepp-Logan. Bottom: Grains. Left: Relative error vs. regularization parameter. Right: Relative error vs. iteration number in Algorithm 1.

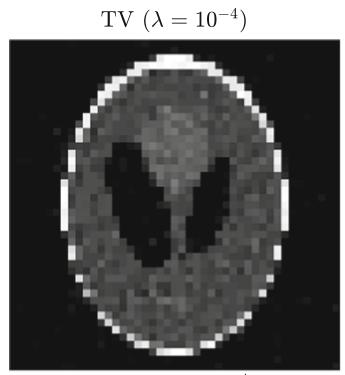

$\operatorname{TV}\left(\lambda=3 \cdot 10^{-4}\right)$

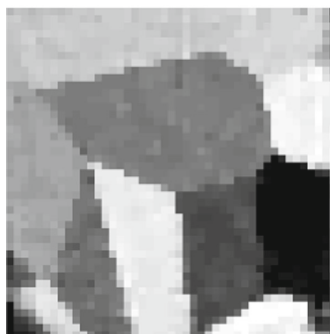

$\operatorname{STV}\left(\lambda=10^{-4}\right)$

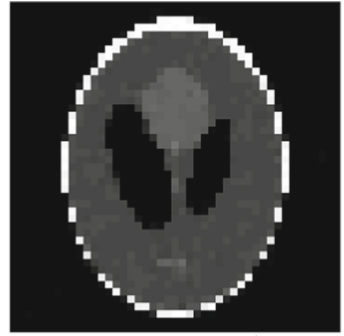

$\operatorname{STV}\left(\lambda=3 \cdot 10^{-4}\right)$

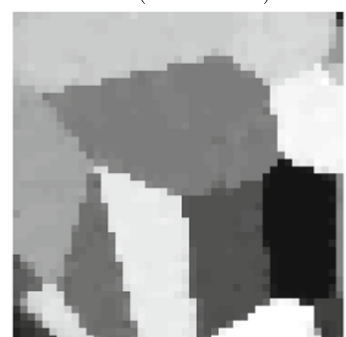

TV-opt $\left(\lambda=10^{-4}\right)$

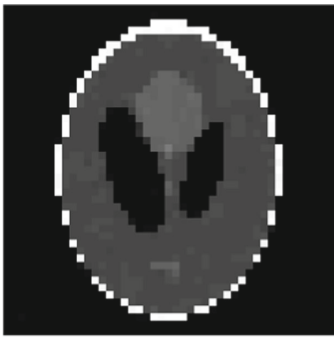

TV-opt $\left(\lambda=3 \cdot 10^{-4}\right)$

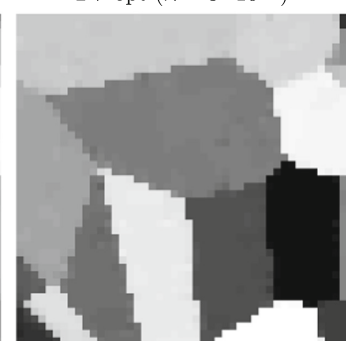

Fig. 4. Reconstructions for $N=45$ in Table 1. Top: Shepp-Logan. Bottom: Grains. 


\subsection{The Larger Example}

We also compute a larger example according to the parameters in Table 1 . In Fig. 5 we show a zoomed part of the expected view angles $\hat{\boldsymbol{\theta}}$ as well as a realization of $\pi_{\text {angles }}(\boldsymbol{\theta})$ that is used to generate the data. In Fig. 6 we show the plots of the relative errors with different choices of $\lambda$ and along the iterations in our method. In this case the difference between $\mathbf{x}_{\mathrm{TV}}$ from solving (12) and $\mathbf{x}_{\mathrm{TV} \text {-opt }}$ from solving (13) is not as big as in the small example, and the main reason is that the variance $\delta^{2}$ in the view angles is much smaller. However, we can still clearly see that our method improves the reconstruction quality in terms of relative error. To compare the reconstruction visually, in Fig. 7 we give the reconstruction results from three methods.

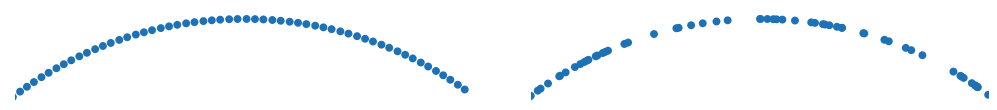

Fig. 5. For the larger experiment in Table 1. Left: the expected view angles $\hat{\boldsymbol{\theta}}$. Right: a realization of $\pi_{\text {angles }}(\boldsymbol{\theta})$. Here we zoomed in on a part of the view angles.
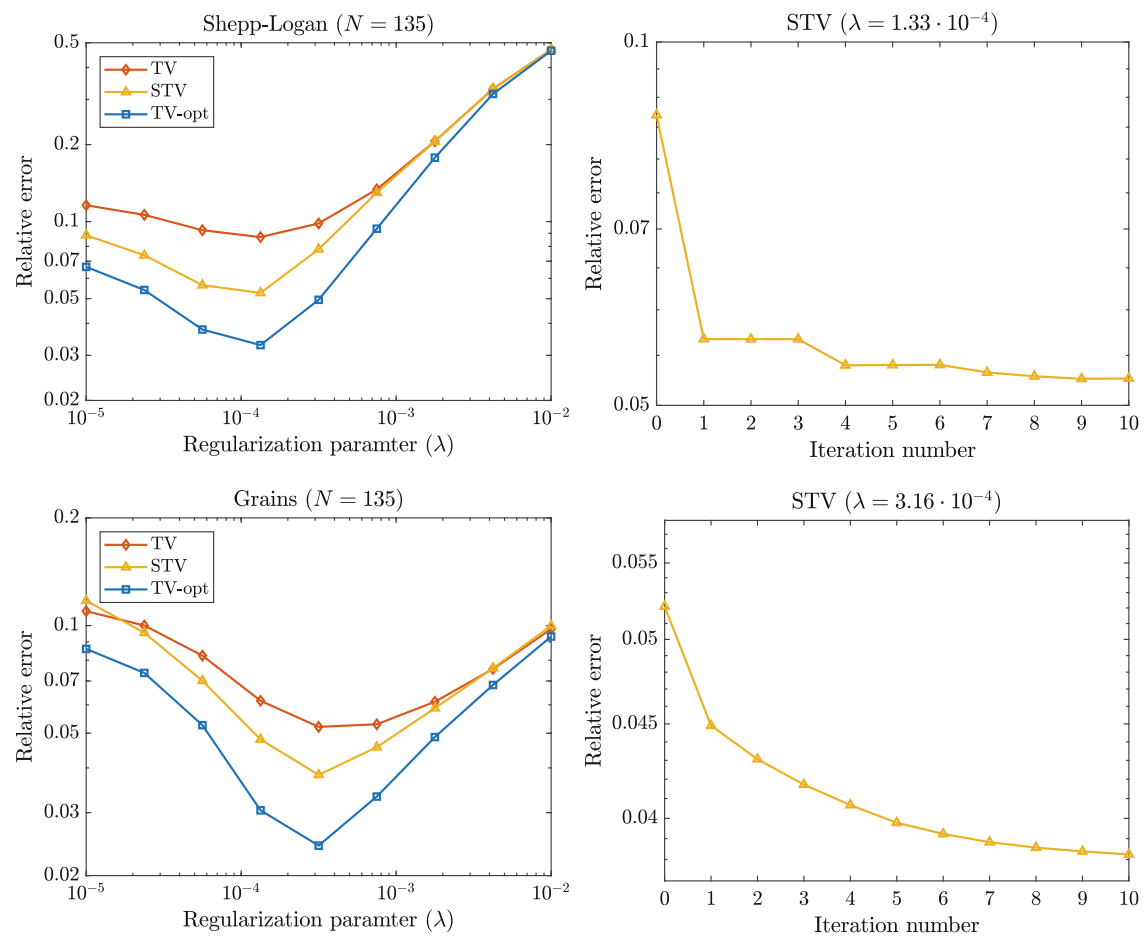

Fig. 6. For the experiment with $N=135$ in Table 1. Top: Shepp-Logan. Bottom: Grains. Left: Relative error vs. regularization parameter. Right: Relative error vs. iteration number in Algorithm 1. 


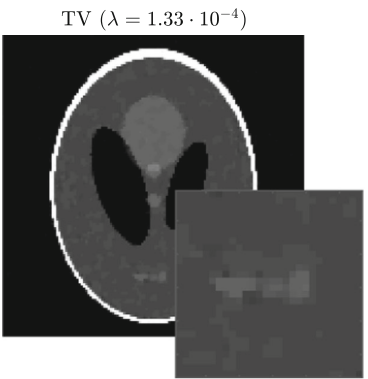

$\operatorname{TV}\left(\lambda=3.16 \cdot 10^{-4}\right)$

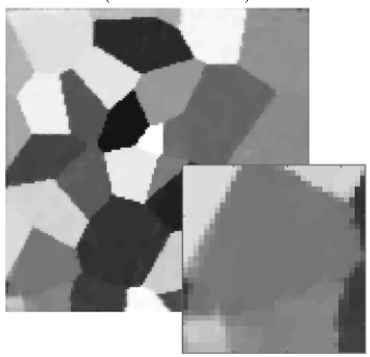

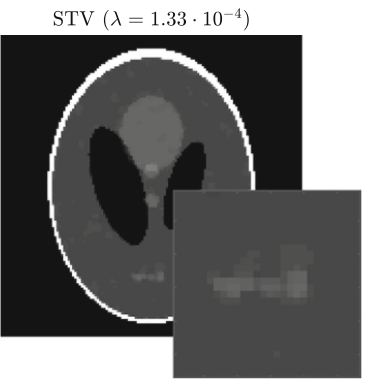

$\operatorname{STV}\left(\lambda=3.16 \cdot 10^{-4}\right)$

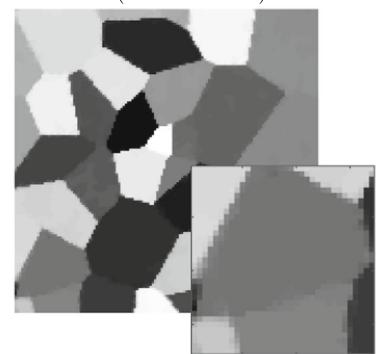

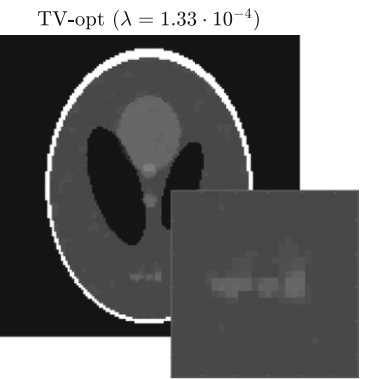

TV-opt $\left(\lambda=3.16 \cdot 10^{-4}\right)$

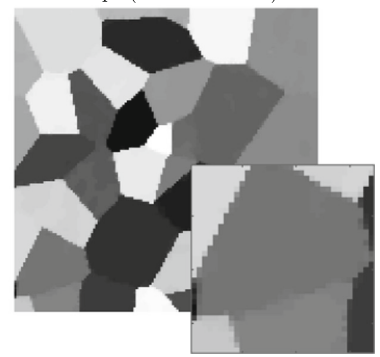

Fig. 7. Reconstructions for $N=135$ in1 Table 1. Top: Shepp-Logan. Bottom: Grains.

Based on our tests, if we increase the image size $n$ and keep the same number of measurements, the quality of the reconstruction by our method gets closer to the one from (12). The reason is that the reconstruction problem becomes more ill-posed and therefore more difficult to deal with. In this case, we would need a better initial guess on $\hat{\mathbf{L}}_{\boldsymbol{\nu} \mid \mathbf{x}}^{[0]}$ and $\boldsymbol{\mu}_{\boldsymbol{\nu} \mid \mathbf{x}}^{[0]}$ in order to obtain a good estimate of $\overline{\mathbf{x}}$. Another idea would be to update the estimate of $\hat{\boldsymbol{\theta}}$ and $\pi_{\text {angles }}(\cdot)$ in each iteration. We leave this to the future study.

\section{Conclusion}

We proposed a new iterative algorithm for the uncertain angle CT problem. The method models the uncertainty of the view angles in the likelihood function. We showed numerically that combining this likelihood with a strong prior such as total variation can significantly improve the relative error and visual quality of reconstructions. Furthermore, we showed a method for approximating the likelihood by a block-diagonal approximation of the covariance, which leads to an algorithm that can run on large-scale CT problems.

\section{References}

1. Natterer, F.: The Mathematics of Computerized Tomography. Wiley, Chicago (1986)

2. Benning, M., Burger, M.: Modern regularization methods for inverse problems. Acta Numerica 27, 1-111 (2018) 
3. Riis, N.A.B., Frøsig, J., Dong, Y., Hansen, P.C.: Limited-data X-ray CT for underwater pipeline inspection. Inverse Prob. 34(3), 034002 (2018)

4. Vandeghinste, B., et al.: Iterative CT reconstruction using shearlet-based regularization. IEEE Tran. Nucl. Sci. 60(5), 3305-3317 (2013)

5. Rudin, L.I., Osher, S., Fatemi, E.: Nonlinear total variation based noise removal algorithms. Physica D 60(1-4), 259-268 (1992)

6. Ferrucci, M., Leach, R.K., Giusca, C., Carmignato, S., Dewulf, W.: Towards geometrical calibration of X-ray computed tomography systems-a review. Measur. Sci. Technol. 26(9), 092003 (2015)

7. Mallick, S.P., Agarwal, S., Kriegman, D.J., Belongie, S.J., Carragher, B., Potter, C.S.: Structure and view estimation for tomographic reconstruction: a bayesian approach. In: Proceedings of the IEEE Computer Society Conference on Computer Vision and Pattern Recognition, vol. 2(1), pp. 2253-2260 (2006). 1641029

8. Fang, Y., Murugappan, S., Ramani, K.: Estimating view parameters from random projections for tomography using spherical MDS. BMC Med. Imaging 10(1), 12 (2010)

9. Basu, S., Bresler, Y.: Uniqueness of tomography with unknown view angles. IEEE Trans. Image Process. 9(6), 1094-1106 (2000)

10. Korolev, Y., Lellmann, J.: Image reconstruction with imperfect forward models and applications in deblurring. SIAM J. Imaging Sci. 11(1), 197-218 (2018)

11. Kaipio, J., Somersalo, E.: Statistical and Computational Inverse Problems. Springer, New York (2005). https://doi.org/10.1007/b138659

12. Madsen, R.B., Hansen, T.M.: Estimation and accounting for the modeling error in probabilistic linearized amplitude variation with offset inversion. Geophysics 83(2), N15-N30 (2018)

13. Hansen, T.M., Cordua, K.S., Holm Jacobsen, B., Mosegaard, K.: Accounting for imperfect forward modeling in geophysical inverse problems exemplified for crosshole tomography. Geophysics 79(3), H1-H21 (2014)

14. Calvetti, D., Dunlop, M., Somersalo, E., Stuart, A.: Iterative updating of model error for bayesian inversion. Inverse Prob. 34(2), 025008 (2018)

15. Kolehmainen, V., Tarvainen, T., Arridge, S.R., Kaipio, J.P.: Marginalization of uninteresting distributed parameters in inverse problems - application to diffuse optical tomography. Int. J. Uncertainty Quantification 1(1), 1-17 (2011)

16. Kennedy, M.C., O'Hagan, A.: Bayesian calibration of computer models. J. Roy. Stat. Soc. Ser. B: Stat. Methodol. 63(3), 425-450 (2001)

17. Kaipio, J., Somersalo, E.: Statistical inverse problems: discretization, model reduction and inverse crimes. J. Comput. Appl. Math. 198(2), 493-504 (2007)

18. Nissinen, A., Heikkinen, L.M., Kaipio, J.P.: The bayesian approximation error approach for electrical impedance tomography - experimental results. Measur. Sci. Technol. 19(1), 015501 (2008)

19. van Aarle, W., et al.: Fast and flexible X-ray tomography using the ASTRA toolbox. Opt. Express 24(22), 25129-25147 (2016). www.astra-toolbox.com

20. van den Berg, E., Friedlander, M.P.: Spot - A Linear-Operator Toolbox: MATLAB software. www.cs.ubc.ca/labs/scl/spot/

21. Hansen, P.C., Jørgensen, J.S.: AIR tools II: algebraic iterative reconstruction methods, improved implementation. Numer. Algorithms 79(1), 107-137 (2017)

22. Chambolle, A., Pock, T.: A first-order primal-dual algorithm for convex problems with applications to imaging. J. Math. Imaging Vis. 40(1), 120-145 (2011) 